Cite this: J. Mater. Chem. A, 2013, 1 13672

Received 23rd May 2013

Accepted 17th August 2013

DOI: 10.1039/c3ta12032e

www.rsc.org/MaterialsA

\section{Oxygen adsorption and dissociation during the oxidation of monolayer $\mathrm{Ti}_{2} \mathrm{C}$}

\begin{abstract}
Li-Yong Gan, ${ }^{a}$ Dan Huang ${ }^{\text {bc }}$ and Udo Schwingenschlögl $\left.\right|^{\star a}$
Exfoliated two-dimensional early transition metal carbides and carbonitrides are usually not terminated by metal atoms but saturated by $\mathrm{O}, \mathrm{OH}$, and/or $\mathrm{F}$, thus making it difficult to understand the surface structure evolution and the induced electronic modifications. To fill this gap, density functional theory and molecular dynamics simulations are performed to capture the initial stage of the oxidation process of $\mathrm{Ti}_{2} \mathrm{C}, \mathrm{a}$ prototypical example from the recently fabricated class of two-dimensional carbides and carbonitrides. It is shown that the unsaturated $\mathrm{Ti} 3 d$ orbitals of the pristine $\mathrm{Ti}_{2} \mathrm{C}$ surface interact strongly with the approaching $\mathrm{O}_{2}$ molecules, resulting in barrierless $\mathrm{O}_{2}$ dissociation. The diffusion of the dissociated $\mathrm{O}$ atoms is also found to be very facile. Molecular dynamics simulations suggest that both dissociation and diffusion are enhanced as the $\mathrm{O}_{2}$ coverage increases to 0.25 monolayer. For a coverage of less than 0.11 monolayer, the adsorbates lead to a minor modification of the electronic properties of $\mathrm{Ti}_{2} \mathrm{C}$, while the modification is remarkable at 0.25 monolayer. The formed $\mathrm{Ti}_{2} \mathrm{CO}_{2}$ after $\mathrm{O}$ saturation is an indirect narrow gap semiconductor $(0.33 \mathrm{eV})$ with high intrinsic carrier concentration at room temperature and high thermodynamic stability at intermediate temperature (e.g., $\left.550{ }^{\circ} \mathrm{C}\right)$.
\end{abstract}

\section{Introduction}

Since the discovery of graphene, ${ }^{\mathbf{1 - 3}}$ tremendous interest has been generated to explore the synthesis, properties, and applications of two-dimensional (2D) materials. ${ }^{4-6}$ Using mechanical $^{7,8}$ or chemical ${ }^{9-11}$ exfoliation, various materials with one or a few atomic layers, such as $\mathrm{BN}, \mathrm{MoS}_{2}, \mathrm{WSe}_{2}, \mathrm{Bi}_{2} \mathrm{Se}_{3}$, and $\mathrm{Bi}_{2} \mathrm{Te}_{3}$, have been fabricated from the weakly bonded layered bulk compounds. These materials exhibit distinctly different properties and therefore can be used in a wide range of applications. ${ }^{\mathbf{1 2 - 1 4}}$ Recently, new families of $2 \mathrm{D}$ early transition metal carbides and carbonitrides, the so-called MXenes $(\mathrm{M}=\mathrm{Ti}, \mathrm{Sr}, \mathrm{V}$, $\mathrm{Cr}$, Ta, Nb, Zr, Mo, Hf, X = C, N or both), have been exfoliated from layered $\mathrm{M}_{n+1} \mathrm{AX}_{n}\left(n=1,2\right.$, and 3), ${ }^{15-17}$ in which A represents elements mainly from groups IIIA and IVA. In $\mathrm{M}_{n+1} \mathrm{AX}_{n}$, the weak chemical A-M bonds allow removal of A ions from the bulk and thus exfoliation. The structural similarity to graphene and high electrical conductivity make these materials promising for electronic device applications, such as lithium ion batteries. ${ }^{18-21}$ It has been demonstrated that the surfaces are covered by $\mathrm{O}, \mathrm{OH}$, and/or $\mathrm{F}$ acceptor groups ${ }^{15}$ and that special electronic and magnetic properties are induced by the surface

\footnotetext{
${ }^{a}$ Physical Sciences and Engineering Division, King Abdullah University of Science and Technology (KAUST), Thuwal 23955-6900, Kingdom of Saudi Arabia. E-mail: udo. schwingenschlogl@kaust.edu.sa; Tel: +966(0)544700080

${ }^{b}$ College of Physics and Technology, Guangxi University, Nanning 530004, People's Republic of China

${ }^{c}$ Department of Physics and Electronic Sciences, Hunan University of Arts and Science, Changde 415000, People's Republic of China
}

adsorbates. Specifically, some MXene monolayers become semiconducting upon $\mathrm{O}$ saturation. ${ }^{22}$

The $\mathrm{O}, \mathrm{OH}$, and/or $\mathrm{F}$ coverage of MXene surfaces leads to difficulties in understanding the surface structure evolution and the induced effects on the electronic properties. To fill this gap, density function theory calculations and molecular dynamics (MD) simulations are performed to study the initial steps of the oxygen adsorption and dissociation processes and the corresponding evolution of the structural and electronic properties. We chose $\mathrm{Ti}_{2} \mathrm{C}$, consisting of three atomic layers $\left(\mathrm{Ti}_{1}-\mathrm{C}-\mathrm{Ti}_{2}\right)$, as a representative case, and have confirmed our results for the five layer case. It is found that both $\mathrm{O}_{2}$ dissociation and $\mathrm{O}$ diffusion are very facile at low coverage. The surface Ti $3 d$ dangling orbitals strongly interact with the approaching $\mathrm{O}_{2}$ molecules, giving rise to a fast dissociation.

\section{Computational details}

This work is based on the Vienna $\mathrm{Ab}$ Initio Simulation Package $^{23-25}$ and the frozen-core projector augmented wave method. $^{26,27}$ The spin polarized Perdew, Burke, and Ernzerhof generalized gradient approximation is employed for the exchange-correlation potential $^{28}$ and a cutoff energy of $500 \mathrm{eV}$ is used for the plane wave expansion. $3 \times 3$ [Fig. 1(a)] and $2 \times 2$ lateral supercells are used together with respective $4 \times 4 \times 1$ and $6 \times 6 \times 1$ Gamma-centered $k$-meshes. A vacuum thickness of $15 \AA$ is introduced to obtain separate monolayers. Moreover, a convergence test for the adsorption energies has been performed with respect to the $k$-point sampling, energy cutoff, and vacuum 


\section{(a)}

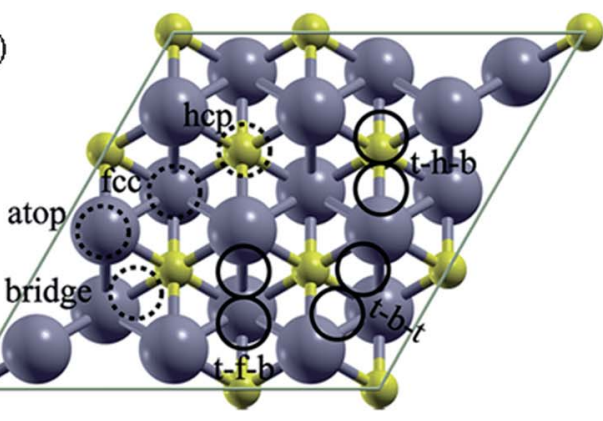

(c)

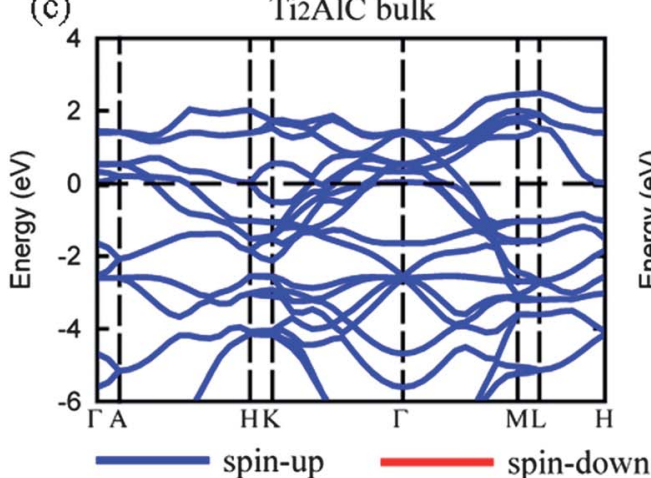

(b)

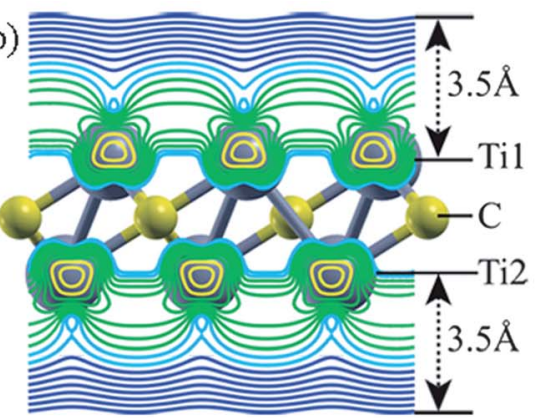

(d)

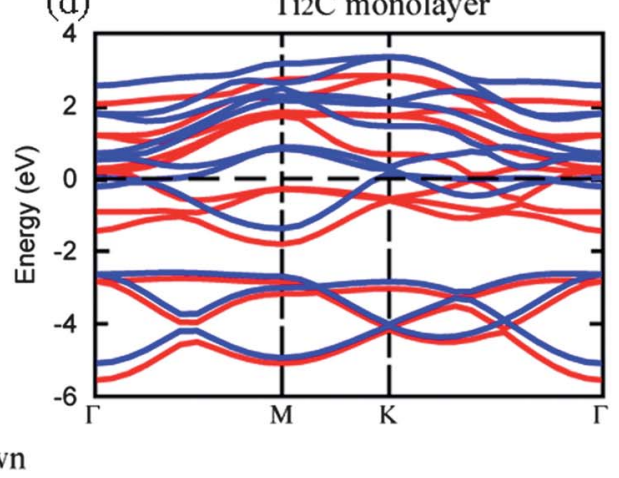

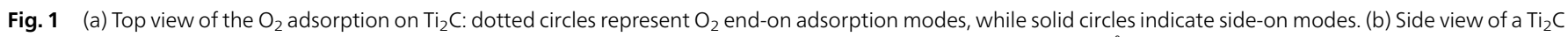

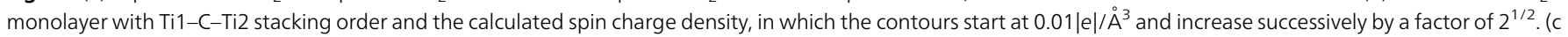
and d) Spin-polarized band structures of bulk $\mathrm{Ti}_{2} \mathrm{AlC}$ and monolayer $\mathrm{Ti}_{2} \mathrm{C}$. The Fermi level is set to zero.

thickness. The structure is fully relaxed until the residual forces are less than $0.02 \mathrm{eV} \AA^{-1}$. A higher energy cutoff, denser $k$-point sampling, and thicker vacuum vary the energy by less than 10 meV. A dipole correction is applied in the asymmetric adsorption configurations..$^{23}$ All the surface adsorbates are added as neutral species. The energy barriers and minimum energy paths (MEPs) of $\mathrm{O}_{2}$ dissociation and oxygen diffusion are computed by the climbing image nudged elastic band (CI-NEB) method. ${ }^{29}$ Five images are used to calculate the reaction path. After a standard transition state search, the transition states are further optimized by the quasi Newton method until the residual forces are less than $0.02 \mathrm{eV}^{-1}$. Ab initio MD simulations are used to investigate the entire $\mathrm{O}_{2}$ dissociation process starting from gas-phase $\mathrm{O}_{2}$ and the thermodynamic stability of the $\mathrm{O}$ saturated $\mathrm{Ti}_{2} \mathrm{C}$ monolayer. The simulations are performed at $823 \mathrm{~K}$ using the Nosé-Hoover thermostat, ${ }^{30}$ and the equations of motion are integrated by the Verlet algorithm ${ }^{31}$ with a time step of $2 \mathrm{fs}$.

\section{Results and discussion}

$\mathrm{Ti}_{2} \mathrm{C}$ has a centered honeycomb structure with $\mathrm{C}$ sandwiched between Ti layers. When a $\mathrm{Ti}_{2} \mathrm{C}$ monolayer is exfoliated from bulk $\mathrm{Ti}_{2} \mathrm{AlC}$, its structure experiences a slight expansion in the (0001) plane and a contraction in the [0001] direction. The nearest neighbor Ti1-Ti1 in-plane bond lengths are $3.066 \AA$ in the bulk and $3.076 \AA$ in $\mathrm{Ti}_{2} \mathrm{C}$, respectively. The distance between the Ti and $\mathrm{C}$ atomic layers shrinks by almost $2 \%$ from the bulk (1.149 $\AA$ ) to the monolayer $(1.128 \AA)$. It is well known that the generalized gradient approximation underestimates the band gaps of semiconductors. ${ }^{32}$ However, since both $\mathrm{Ti}_{2} \mathrm{C}$ and $\mathrm{Ti}_{2} \mathrm{AlC}$ experimentally are metallic, ${ }^{17,20}$ our results do not suffer from this limitation. Fig. 1(c) and (d) show band structures of $\mathrm{Ti}_{2} \mathrm{AlC}$ and $\mathrm{Ti}_{2} \mathrm{C}$, both being metallic, consistent with the experimental situation. The former is non-magnetic with degenerate spin channels, while the latter is magnetic with a total magnetic moment of 1.85 $\mu_{\mathrm{B}}$ per unit cell, which is mainly located at the unsaturated surface Ti atoms. The spin up and spin down channels are split but both cross the Fermi level. The metallicity of $\mathrm{Ti}_{2} \mathrm{C}$ makes it potentially applicable in electrical devices. Spin charge density contours of $\mathrm{Ti}_{2} \mathrm{C}$ imply that the induced magnetic moment mainly originates from surface Ti $3 d$ dangling orbitals, similar to the case of $\mathrm{Ti}_{3} \mathrm{C}_{2}{ }^{18}$ Furthermore, the spin charge density plot shows that the surface charge extends to the vacuum up to $3.5 \AA$. We will demonstrate later that this extension could facilitate charge transfer from the surface to an $\mathrm{O}_{2}$ molecule and thus enhancing the $\mathrm{O}_{2}$ dissociation.

As a starting point, we first investigate $\mathrm{O}_{2}$ adsorption on $\mathrm{Ti}_{2} \mathrm{C}$. There are two typical adsorption configurations for an oxygen molecule, the side-on and the end-on mode, ${ }^{33}$ which are depicted in Fig. 1(a). We have performed simulations with one $\mathrm{O}_{2}$ on one side of a $3 \times 3$ supercell, corresponding to a coverage of 0.11 monolayers (MLs). These adsorbed precursors will be used as possible initial states for the subsequent energy barrier and minimum energy path calculations by the climbing-image nudged elastic band method. ${ }^{29}$ The calculated $\mathrm{O}-\mathrm{O}$ bond length and frequency in gas phase $\mathrm{O}_{2}$ are $1.233 \AA$ and $1559 \mathrm{~cm}^{-1}$, respectively, in good agreement with the experimental values ${ }^{34}$ of $1.207 \AA$ and $1555 \mathrm{~cm}^{-1}$ and previous theoretical values of $1.235 \AA$ and $1558 \mathrm{~cm}^{-1}$. $^{35}$ 
Table 1 Adsorption energy $E_{\mathrm{ad}}(\mathrm{eV})$, bond length $d_{\mathrm{O} 1-\mathrm{O} 2}(\AA)$, vibrational frequency $f\left(\mathrm{~cm}^{-1}\right)$, Bader charge $Q(|e|)$, and magnetic moment $M\left(\mu_{\mathrm{B}}\right)$ of the adsorbed diatomic oxygen. The adsorption sites are defined in Fig. 1. In the end-on modes, $\mathrm{O} 1$ is bonded to the surface. The values in brackets refer to an $\mathrm{O}_{2}$ coverage of $0.25 \mathrm{ML}$, i.e., one $\mathrm{O}_{2}$ in a $2 \times 2$ supercell

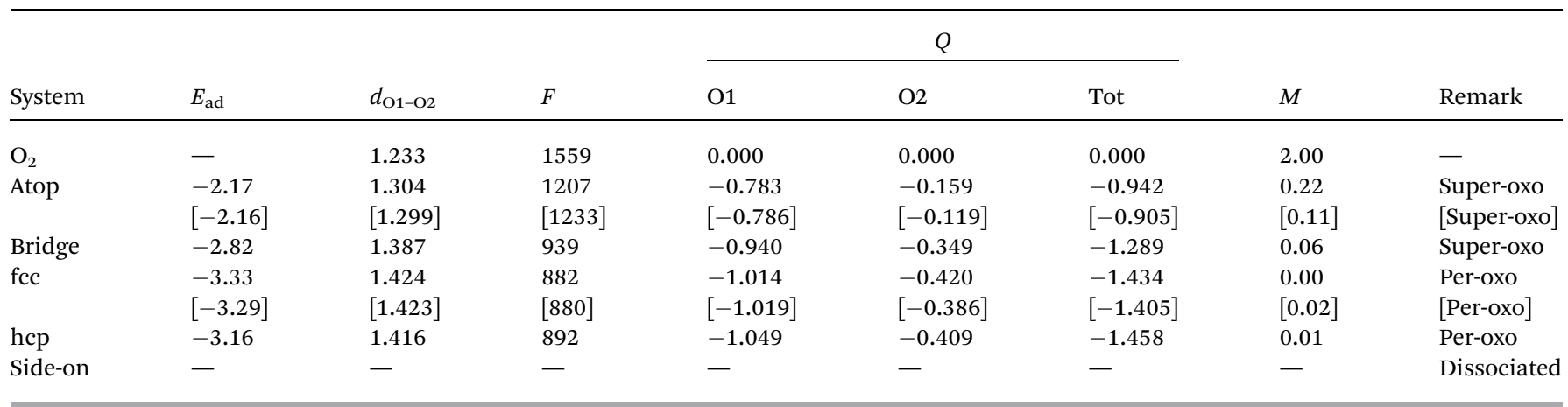

Table 1 summarizes the energetic, structural, and electronic results of $\mathrm{O}_{2}$ adsorption on $\mathrm{Ti}_{2} \mathrm{C}$ in different configurations. Interestingly, when $\mathrm{O}_{2}$ molecules were placed in the three sideon modes, they would dissociate spontaneously after full structural relaxation, suggesting that it is unlikely to detect $\mathrm{O}_{2}$ adsorption in side-on modes on $\mathrm{Ti}_{2} \mathrm{C}$. As for the end-on modes, the stability follows this order: atop $(-2.17 \mathrm{eV})<$ bridge $(-2.82$ $\mathrm{eV})<\mathrm{hcp}(-3.16 \mathrm{eV})<\mathrm{fcc}(-3.33 \mathrm{eV})$. These values are much lower than theoretical values on transition metals, ${ }^{36,37}$ suggesting that $\mathrm{Ti}_{2} \mathrm{C}$ is more susceptible to $\mathrm{O}_{2}$ adsorption. As the coordination increases from the atop to fcc/hcp site, the $\mathrm{O}-\mathrm{O}$ bond strength weakens gradually. The predicted bond lengths are $1.304 \AA$, $1.387 \AA$, $1.416 \AA$, and $1.424 \AA$, and the corresponding vibrational frequencies are $1207 \mathrm{~cm}^{-1}, 939 \mathrm{~cm}^{-1}, 892$ $\mathrm{cm}^{-1}$, and $882 \mathrm{~cm}^{-1}$. The trend is similar to that for the adsorption of other diatomic molecules (e.g., CO or NO) on transition metal surfaces ${ }^{36,37}$ because increasing the coordination facilitates charge transfer from the metal surface to the molecular anti-bonding states. Bader charge analysis ${ }^{38}$ indicates that the total charge of the adsorbed $\mathrm{O}_{2}$ increases from atop to fcc/hcp. Meanwhile, there are no residual magnetic moments as $\mathrm{O}_{2}$ is adsorbed at the two three-fold sites. According to these results, $\mathrm{O}_{2}$ adsorption at the atop and bridge sites can be classified as super-oxo species and at the two three-fold sites as peroxo species. ${ }^{39}$
Before investigating the oxygen dissociation mechanism on monolayer $\mathrm{Ti}_{2} \mathrm{C}$, it is necessary to study the relative stability of monoatomic oxygen species. It is found that oxygen adsorption at the fcc site is energetically favorable. Fig. 2 shows the charge redistribution in this case. It is clear that the charge depletes from the surface $\mathrm{Ti}$ atoms and accumulates around the $\mathrm{O}$ atom. The Bader analysis ${ }^{38}$ shows that monoatomic oxygen gains an effective charge of $-1.465|e|$, which is very close to the values obtained for oxygen anions in bulk transition oxides (e.g., $\left.\mathrm{LaMnO}_{3}\right) .{ }^{39}$ Additionally, no charge accumulation can be seen in the $\mathrm{O}-\mathrm{Ti}$ bonding region, indicative of an ionic bond between $\mathrm{O}$ and Ti. Therefore, it can be concluded that the adsorbed monoatomic $\mathrm{O}$ species on $\mathrm{Ti}_{2} \mathrm{C}$ is $\mathrm{O}^{2-}$. Subsequently, configurations with adsorption of two $\mathrm{O}^{2-}$ ions at adjacent fcc sites are adopted as the final state to map out the MEPs for $\mathrm{O}_{2}$ dissociation. The least (atop) and most (fcc) stable configurations for $\mathrm{O}_{2}$ adsorption are selected as representative initial states, and the CI-NEB method ${ }^{29}$ is used to locate the transition states. The two MEPs are addressed in Fig. 3, where arbitrary intermediate states (i) and (iv) connecting the initial and final states are shown. The process from the most stable initial state (fcc) is exothermic by $7.38 \mathrm{eV}$ without any barrier. The $\mathrm{O}_{1}-\mathrm{O}_{2}$ distance is elongated from $1.442 \AA$ (per-oxo-like adsorbed $\mathrm{O}_{2}$ ) to $3.093 \AA$ (dissociated). From the atop site adsorption configuration the process is exothermic by $8.54 \mathrm{eV}$ and no well-defined transition

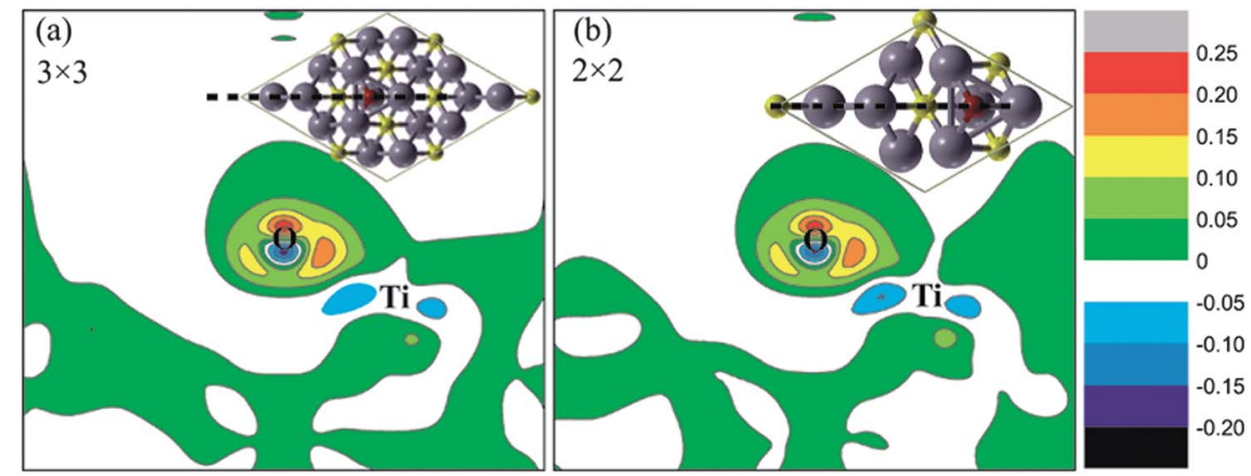

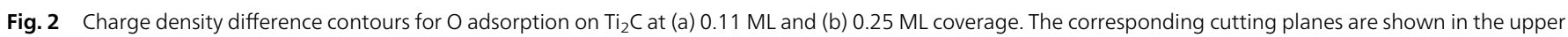
right corners. 


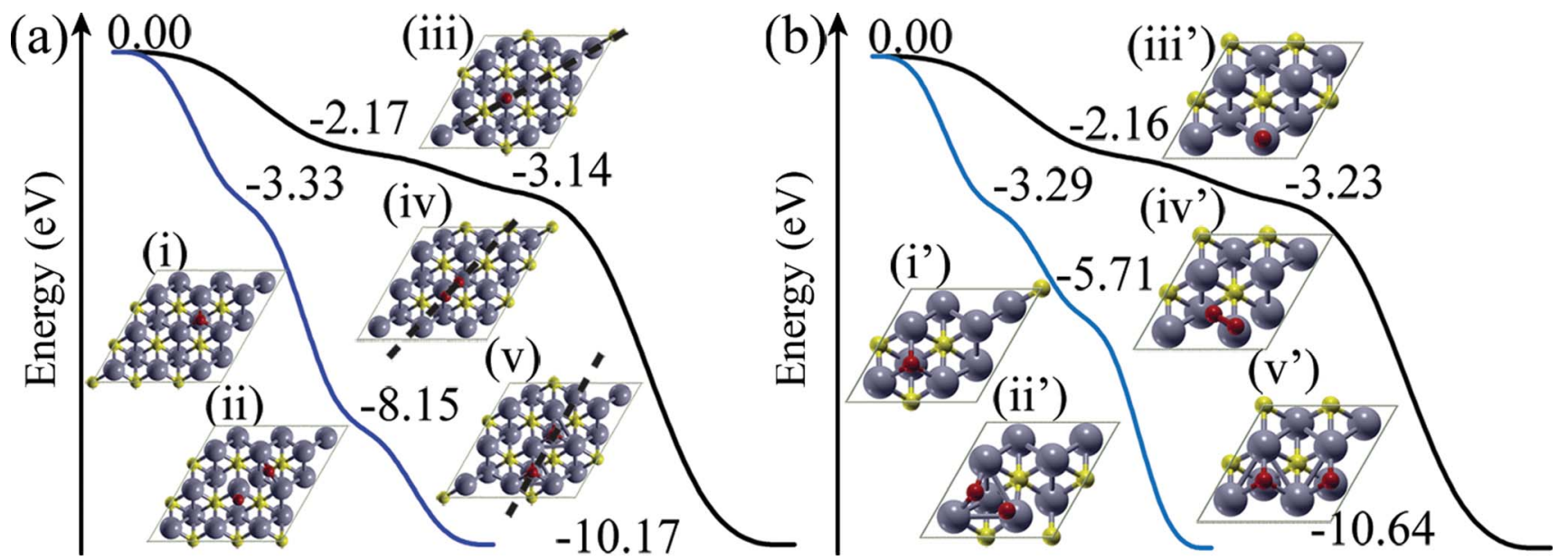

Fig. 3 MEPs for $\mathrm{O}_{2}$ dissociation on monolayer $\mathrm{Ti}_{2} \mathrm{C}$ in a (a) $3 \times 3$ and (b) $2 \times 2$ supercell from the fcc site [blue curve, (i), (i') $\rightarrow$ (ii), (ii') $\left.\rightarrow(\mathrm{v}),\left(\mathrm{v}^{\prime}\right)\right]$ and atop site [black curve (iii), (iii') $\rightarrow$ (iv), (iv') $\rightarrow(v),\left(v^{\prime}\right)$ ] adsorption configurations. The energy of gas phase $\mathrm{O}_{2}$ is set to zero. The black dashed lines in (iii), (iv), and (v) show the cutting planes of the charge density difference contours in Fig. 6.

state is obtained. The two MEPs show that $\mathrm{O}_{2}$ decomposition from both the least and most stable configurations is very facile, suggesting that $\mathrm{O}_{2}$ dissociation on monolayer $\mathrm{Ti}_{2} \mathrm{C}$ takes place very fast without any detectable barrier.

Subsequently, the $\mathrm{O}^{2-}$ diffusion between two adjacent fcc sites is analyzed to address the $\mathrm{O}^{2-}$ migration on $\mathrm{Ti}_{2} \mathrm{C}$, as shown in Fig. 4. The oxide ion starts from the first fcc site, passes a bridge site with a modest barrier of $0.53 \mathrm{eV}$ to reach an hcp site, and then overcomes another barrier of $0.26 \mathrm{eV}$ to get to the second fcc site. Additionally, in the entire process, the charge of the monoatomic oxygen does hardly change: fcc1 $(-1.465|e|)$ $\rightarrow$ bridge $(-1.416|e|) \rightarrow \operatorname{hcp}(-1.492|e|) \rightarrow \operatorname{bridge}(-1.416|e|)$ $\rightarrow$ fcc $2(-1.465|e|)$. Therefore, as demonstrated above, we are confronted with $\mathrm{O}^{2-}$ diffusion, and the process is very facile on $\mathrm{Ti}_{2} \mathrm{C}$. The barrierless dissociation of $\mathrm{O}_{2}$ and the fast $\mathrm{O}$ diffusion, from another point of view, explain why the surface is usually found to be saturated by $\mathrm{O}$ or other groups. ${ }^{15}$

Subsequently, MD simulations are used to investigate the entire dissociation process from the gas-phase $\mathrm{O}_{2}$. An $\mathrm{O}_{2}$

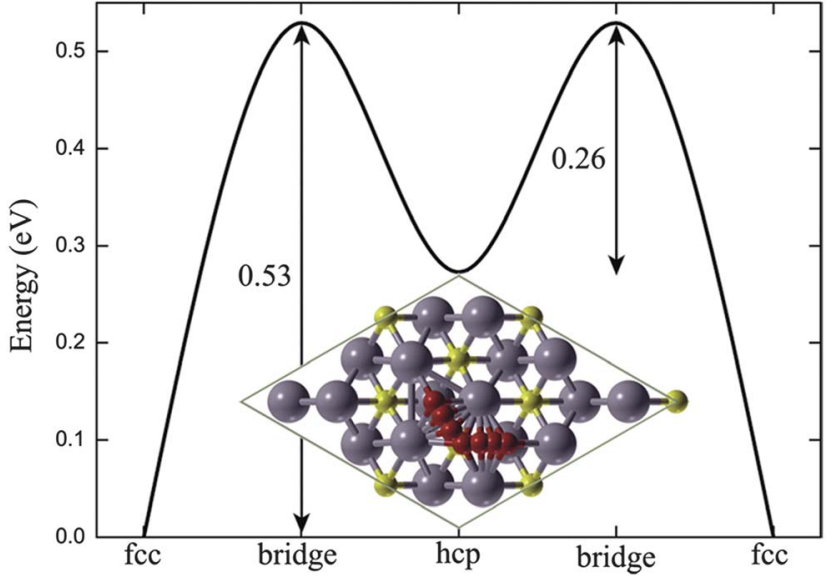

Fig. 4 MEP for $\mathrm{O}^{2-}$ diffusion between two adjacent fcc sites. The energy at the fcc sites is set to zero. molecule is initially placed above the atop or fcc site at approximately $4 \AA$. Only the results for the first case are shown in Fig. 5 because the overall behaviors in the entire process are similar for both cases. The free $\mathrm{O}_{2}$ molecule is firstly adsorbed at the atop site with a slightly tilted bond axis, forming a superoxo species $\left(d_{\mathrm{O} 1-\mathrm{O} 2}=1.371 \AA\right)$ at 278 fs. Then, the $\mathrm{O}-\mathrm{O}$ bond is progressively elongated and starts to split at about $360 \mathrm{fs}$. Next, the two $\mathrm{O}^{2-}$ ions diffuse and finally evolve into their most stable adsorption configuration (each $\mathrm{O}^{2-}$ at an fcc site) at $492 \mathrm{fs}$. After dissociation, the MD energy profile shows an almost periodic oscillation (loops of about $230 \mathrm{fs}$ ). This fact is ascribed to a repeated diffusion of the two $\mathrm{O}^{2-}$ ions between the most stable

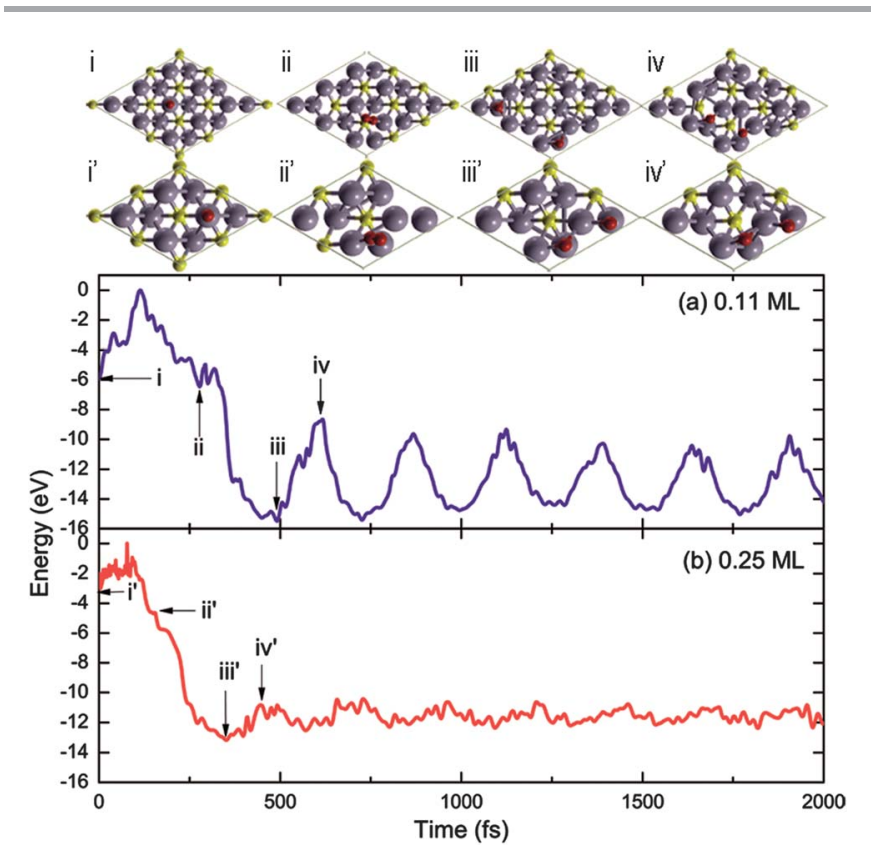

Fig. 5 Energy profiles from $M D$ simulations for $\mathrm{O}_{2}$ dissociation on $\mathrm{Ti}_{2} \mathrm{C}$ at (a) 0.11 $\mathrm{ML}$ and (b) $0.25 \mathrm{ML}$ coverage. Insets i to iv $(0.11 \mathrm{ML})$ and $\mathrm{i}^{\prime}$ to iv' $(0.25 \mathrm{ML})$ give top views of the initial set, the superoxide as well as the most (at two fcc sites) and least (at two bridge sites) stable configurations of the two dissociated $\mathrm{O}^{2-}$ ions. 
adsorption configurations. Though the barrier in each loop seems to be very high, the diffusion repeats as time goes on, implying that it can proceed easily at this temperature (i.e., $550{ }^{\circ} \mathrm{C}$ ), consistent with the CI-NEB results.

When $\mathrm{O}_{2}$ molecules are placed in the three side-on modes they dissociate spontaneously after structural relaxation. As a matter of fact, the tilted adsorption configuration found in our MD simulations [inset (ii) in Fig. 5] is not of the pure end-on atop-site type but a combination of end-on and side-on configurations. The sideon contribution subsequently promotes direct $\mathrm{O}_{2}$ dissociation starting from an off-atop site instead of indirect dissociation after diffusion to an fcc site, suggesting that it is more likely that $\mathrm{O}_{2}$ dissociation follows the path from the super-oxo species, close to the path of Fig. 3(iii)-(v). Therefore, projected densities of states and corresponding charge density difference contours along this path are calculated to obtain further insight into the dissociation process on $\mathrm{Ti}_{2} \mathrm{C}$. First, $\mathrm{O}_{2}$ is fixed above the surface at $2.5 \AA$ [Fig. 6(a) and (e)]. At this height it already interacts with the surface. All the molecular orbitals of $\mathrm{O}_{2}$ shift down relative to the Fermi level, especially the empty $\pi_{\mathrm{g}}^{*}$ anti-bonding orbital. Charge accumulation affects this state and the bond accordingly is weakened. In fact, if the height was not fixed, it would relax spontaneously to the atop site adsorption configuration. As the $\mathrm{O}_{2}$ dissociation proceeds, the molecular orbitals near the Fermi level

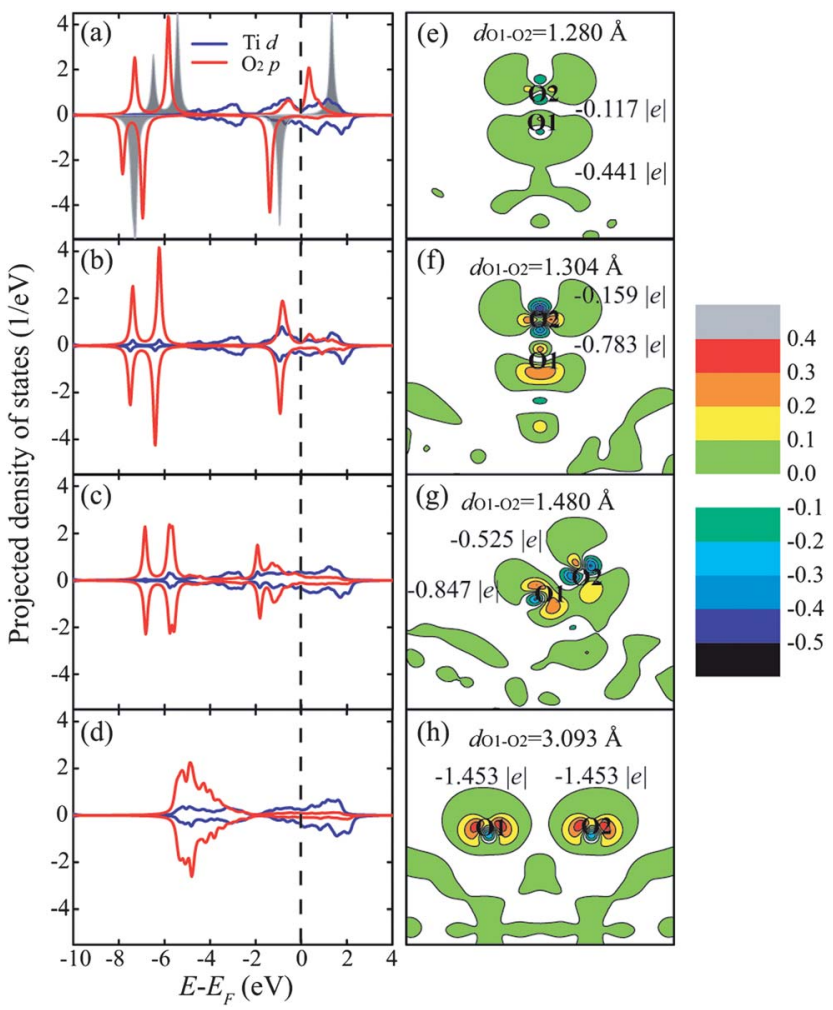

Fig. 6 (a)-(d) Projected density of states and (e)-(h) charge density difference for the oxygen dissociation on $\mathrm{Ti}_{2} \mathrm{C}$ via the atop site pathway: (a and e) $\mathrm{O}_{2}$ above a $\mathrm{Ti}$ atop site at a height of $2.5 \AA$. (b and f) Stable $\mathrm{O}_{2}$ atop site adsorption. (c and $\mathrm{g}$ ) Representative intermediate state, see Fig. 3(d), connecting the initial and final states. ( $d$ and $h$ ) Final state of $\mathrm{O}_{2}$ dissociation. The corresponding cutting planes are shown in Fig. 3(iii)-(v). The projected density of states of a free $\mathrm{O}_{2}$ molecule (shaded area) is shown as a reference. continue shifting downward and eventually all the molecular orbitals merge into one main state, as shown in Fig. 6(b)-(d). During this process, charge accumulation in the $\pi_{\mathrm{g}}^{*}$ orbital and depletion of the $\sigma$-like bonding states become more and more evident, and the total charge of $\mathrm{O}_{2}$ becomes more and more negative, see Fig. 6(f) and (g). Finally, the $\mathrm{O}-\mathrm{O}$ bonding states disappear and O-Ti bonds emerge instead [Fig. 6(h)]. Thus, two $\mathrm{O}^{2-}$ ions are formed, each with an effective charge of $-1.453|e|$. These results verify that a neutral $\mathrm{O}_{2}$ molecule ultimately splits into two $\mathrm{O}^{2-}$ ions on the $\mathrm{Ti}_{2} \mathrm{C}$ monolayer. During this process, the main features of the Ti $3 d$ states remain intact, in particular the gap around $-2 \mathrm{eV}$. However, the charge transfer from Ti to O leads to a reduction of the $\mathrm{Ti} 3 d$ density of states around the Fermi level.

In addition, the dissociation of $\mathrm{O}_{2}$ is simulated in a $2 \times 2$ supercell $(0.25 \mathrm{ML})$ to investigate possible coverage effects. Only $\mathrm{O}_{2}$ adsorption at the least (atop) and most (fcc) stable sites (see the values in brackets in Table 1) was considered for calculating the $\mathrm{O}_{2}$ dissociation. As the $\mathrm{O}_{2}$ coverage increases from $0.11 \mathrm{ML}$ to $0.25 \mathrm{ML}$, the adsorption results do not alter remarkably. For the two configurations, the MEPs [Fig. 3(b)] are almost the same as those in the $3 \times 3$ supercell, and again no barriers were found. In the $\mathrm{O}^{2-}$ diffusion process, the two calculated barriers are $0.58 \mathrm{eV}$ and $0.29 \mathrm{eV}$, i.e., only $0.05 \mathrm{eV}$ and $0.03 \mathrm{eV}$ higher than those in the $0.11 \mathrm{ML}$ case. The charge density difference plots for $\mathrm{O}^{2-}$ adsorption in the $3 \times 3$ and $2 \times 2$ [Fig. 2(b)] supercells are also very similar, both indicating an ionic bond between $\mathrm{O}$ and Ti. Fig. 5(b) shows the MD results for the $2 \times 2$ supercell. Generally, the main features of the dissociation of $\mathrm{O}_{2}$ and the diffusion of $\mathrm{O}^{2-}$ (the almost periodic oscillation) resemble each other. The configurations ( $\left.\mathrm{iii}^{\prime}\right)$ and (vi') in Fig. 5 almost reproduce the final state of the $\mathrm{O}_{2}$ dissociation $\left(\mathrm{O}^{2-}\right.$ at fcc sites $)$ and the transition state of the $\mathrm{O}^{2-}$ diffusion in the CI-NEB simulations. Besides, it can be seen that less time is required for a free $\mathrm{O}_{2}$ molecule to dissociate at 0.25 ML (352 fs versus $492 \mathrm{fs}$ ). The barriers ascribed to the $\mathrm{O}^{2-}$ diffusion are much lower than

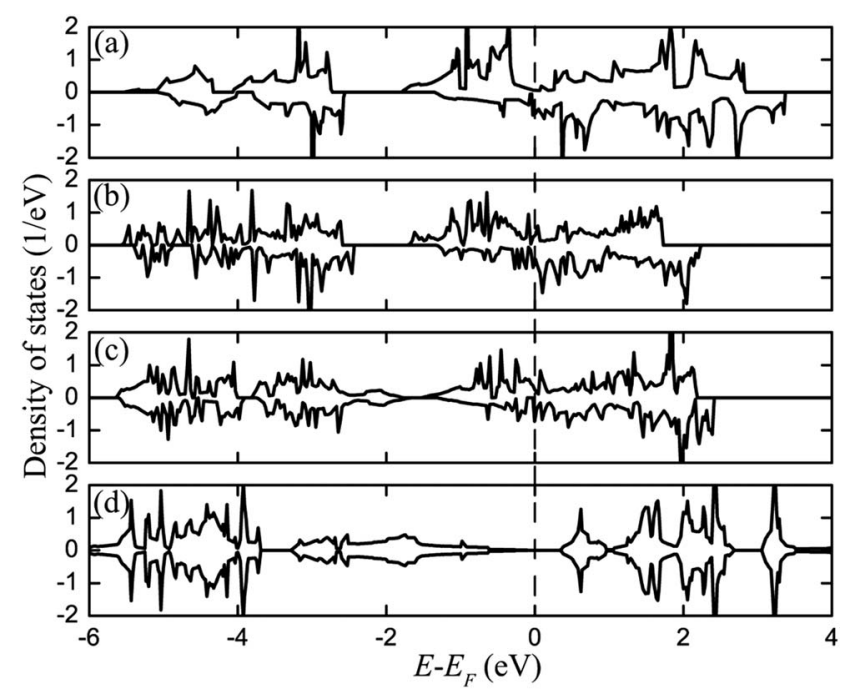

Fig. 7 Density of states (per atom) for (a) pristine $\mathrm{Ti}_{2} \mathrm{C}$ as well as for $\mathrm{O}$ coverage of (b) $0.11 \mathrm{ML}$, (c) $0.25 \mathrm{ML}$, and (d) $1.00 \mathrm{ML}$. 

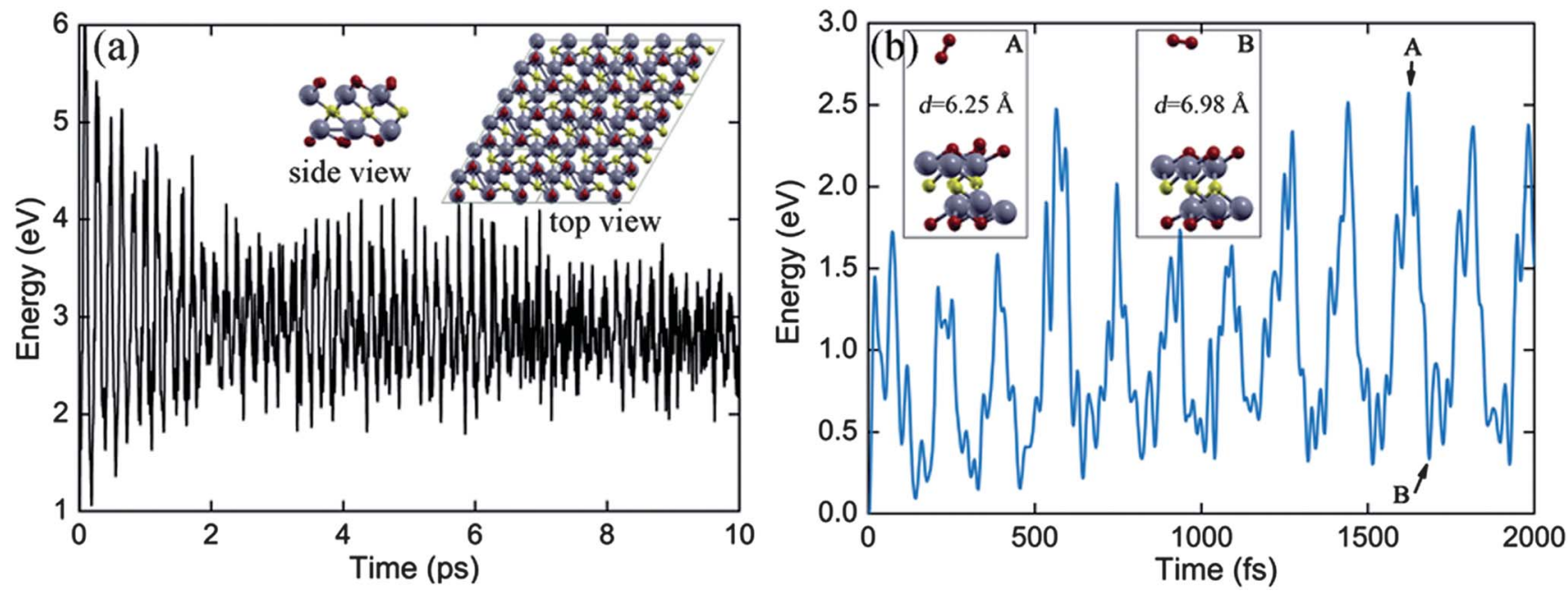

Fig. 8 Energy profiles from MD simulations for $(\mathrm{a}) \mathrm{Ti}_{2} \mathrm{CO}_{2}$ and (b) with one additional oxygen molecule on $\mathrm{Ti}_{2} \mathrm{CO}_{2}$. The insets in (a) show the side and top views after 10 ps. Structures $A$ and $B$ in (b) indicate the least and most stable configurations.

those at $0.11 \mathrm{ML}$. This may be due to the fact that the synergetic effects between $\mathrm{O}^{2-}$ ions are more evident at 0.25 ML. Moreover, MD simulations for $\mathrm{O}_{2}$ dissociation on $\mathrm{Ti}_{2} \mathrm{C}$ in a $2 \times 2$ supercell starting from $\mathrm{O}_{2}$ above an fcc site show that the main features are similar to the case of $\mathrm{O}_{2}$ above an atop site [Fig. 5(b) and inset (ii')], indicating that different initial configurations will not affect the results: $\mathrm{O}_{2}$ will first adsorb at an off-atop site with the $\mathrm{O}-\mathrm{O}$ bond tilted. Our results manifest that increasing the coverage to $0.25 \mathrm{ML}$ facilitates both the oxygen dissociation and $\mathrm{O}^{2-}$ diffusion.

Densities of states obtained for pristine $\mathrm{Ti}_{2} \mathrm{C}$ as well as for $\mathrm{O}$ coverage of $0.11 \mathrm{ML}, 0.25 \mathrm{ML}$, and 1.00 ML on both sides are shown in Fig. 7 to obtain insight into the evolution of the electronic properties during the saturation process. At a coverage of $0.11 \mathrm{ML}$, the adsorbed $\mathrm{O}$ atoms lead to minor modifications of the band features of pristine $\mathrm{Ti}_{2} \mathrm{C}$, introducing a slight energetical upshift of the states relative to the Fermi level. As the coverage increases to $0.25 \mathrm{ML}$, the bands shift further upwards and the gap ${ }^{\mathbf{2 1}}$ in the occupied states is remarkably narrowed. When the $\mathrm{Ti}_{2} \mathrm{C}$ monolayer is saturated by $\mathrm{O}, \mathrm{Ti}_{2} \mathrm{CO}_{2}$ is formed with $\mathrm{O}$ atoms at the most stable adsorption sites (fcc) on both surfaces. The resulting structure has been described in ref. 40 and is demonstrated in the inset of Fig. 8(a). In $\mathrm{Ti}_{2} \mathrm{CO}_{2}$ the gap in the occupied states finally is located at the Fermi level and a semiconducting character is obtained with degenerate spin channels. It is found that $\mathrm{Ti}_{2} \mathrm{CO}_{2}$ is a narrow gap semiconductor with an indirect band gap of $0.36 \mathrm{eV}$. The estimated anisotropic electron and hole effective masses of $\mathrm{Ti}_{2} \mathrm{CO}_{2}$ are $0.135-0.190 m_{0}$ and $0.181-0.200 m_{0}\left(m_{0}\right.$ being the mass of an electron), respectively, yielding an intrinsic carrier density of 5.97-9.82 $\times 10^{14} \mathrm{~cm}^{-3}$ at room temperature $(300 \mathrm{~K})$. These values verify that the monolayer maintains a good electronic conductivity. Additionally, each oxygen atom gains an effective charge of $-1.312|e|$, which is still very close to the value of an oxygen anion in bulk transition metal oxides. ${ }^{39}$

Previous experimental studies indicate that early transition carbide catalysts, such as WC nanoparticles, are not stable but form $\mathrm{WO}_{x}$ in an oxidizing environment. ${ }^{41-43}$ Therefore, it is necessary to explore the thermodynamic stability of $\mathrm{Ti}_{2} \mathrm{C}$ in an oxidizing environment. We have carried out MD simulations for oxygen saturated $\mathrm{Ti}_{2} \mathrm{C}$ (i.e., $\mathrm{Ti}_{2} \mathrm{CO}_{2}$ ) at an intermediate temperature of $823 \mathrm{~K}$ in a $2 \times 3$ supercell. As shown in Fig. 8(a), the structure does not disrupt in a 10 ps simulation. Also, MD simulations with an additional $\mathrm{O}_{2}$ placed above the $\mathrm{Ti}_{2} \mathrm{CO}_{2}$ surface at a height of $\sim 2.5 \AA$ have been performed [Fig. 8(b)]. It can be seen that the $\mathrm{O}_{2}$ is repelled back into the vacuum and oscillates between configurations with the $\mathrm{O}-\mathrm{O}$ bonds almost parallel and perpendicular to the surface. This suggests that $\mathrm{Ti}_{2} \mathrm{CO}_{2}$ is highly resistant against further oxidation to $\mathrm{TiO}_{2}$ at such a temperature, allowing its stable utilization.

\section{Conclusions}

A combination of density functional theory and MD calculations is employed to capture the fast $\mathrm{O}_{2}$ adsorption and dissociation processes on monolayer $\mathrm{Ti}_{2} \mathrm{C}$, which is experimentally always saturated by various chemical groups. It is found that $\mathrm{O}_{2}$ dissociates without any barrier and the MD simulations suggest that the dissociation starts from the super-oxo species. The fast dissociation process is ascribed to the unsaturated $\mathrm{Ti} 3 d$ orbitals. The diffusion of the dissociated $\mathrm{O}$ atoms is also very facile with a maximum barrier height of $0.53 \mathrm{eV}$ at an $\mathrm{O}_{2}$ coverage of $0.11 \mathrm{ML}$. As the $\mathrm{O}_{2}$ coverage increases to $0.25 \mathrm{ML}$, both the $\mathrm{O}_{2}$ dissociation and $\mathrm{O}$ diffusion are accelerated. Further analysis shows that the adsorbed $\mathrm{O}$ alters the energy bands only slightly at an O coverage lower than $0.11 \mathrm{ML}$, while the characteristic energy gap within the occupied Ti $3 d$ states shifts remarkably upwards and becomes narrower at 0.25 ML. When the system is $\mathrm{O}$ saturated the gap appears at the Fermi level and induces a semiconducting character. The formed $\mathrm{Ti}_{2} \mathrm{CO}_{2}$ monolayer exhibits a high carrier concentration at room temperature as well as a high thermodynamic stability at intermediate temperature. 


\section{References}

1 K. S. Novoselov, A. K. Geim, S. V. Morozov, D. Jiang, Y. Zhang, S. V. Dubonos, I. V. Grigorieva and A. A. Firsov, Science, 2004, 306, 666.

2 K. S. Novoselov, A. K. Geim, S. V. Morozov, D. Jiang, M. I. Katsnelson, I. V. Grigorieva, S. V. Dubonos and A. A. Firsov, Nature, 2005, 438, 197.

3 Y. Zhang, Y.-W. Tan, H. L. Stormer and P. Kim, Nature, 2005, 438, 201.

4 C. Lee, Q. Li, W. Kalb, X.-Z. Liu, H. Berger, R. W. Carpick and J. Hone, Science, 2010, 328, 76.

5 F. Schwierz, Nat. Nanotechnol., 2010, 5, 487.

6 L. Britnell, R. V. Gorbachev, R. Jalil, B. D. Belle, F. Schedin, A. Mishchenko, T. Georgiou, M. I. Katsnelson, L. Eaves, S. V. Morozov, N. M. R. Peres, J. Leist, A. K. Geim, K. S. Novoselov and L. A. Ponomarenko, Science, 2012, 335, 947.

7 K. S. Novoselov, D. Jiang, F. Schedin, T. J. Booth, V. V. Khotkevich, S. V. Morozov and A. K. Geim, Proc. Natl. Acad. Sci. U. S. A., 2005, 102, 10451.

8 B. Radisavljevic, A. Radenovic, J. Brivio, V. Giacometti and A. Kis, Nat. Nanotechnol., 2011, 6, 147.

9 J. N. Coleman, M. Lotya, A. O'Neill, S. D. Bergin, P. J. King, U. Khan, K. Young, A. Gaucher, S. De, R. J. Smith, I. V. Shvets, S. K. Arora, G. Stanton, H.-Y. Kim, K. Lee, G. T. Kim, G. S. Duesberg, T. Hallam, J. J. Boland, J. J. Wang, J. F. Donegan, J. C. Grunlan, G. Moriarty, A. Shmeliov, R. J. Nicholls, J. M. Perkins, E. M. Grieveson, K. Theuwissen, D. W. McComb, P. D. Nellist and V. Nicolosi, Science, 2011, 331, 568.

10 R. J. Smith, P. J. King, M. Lotya, C. Wirtz, U. Khan, S. De, A. O'Neill, G. S. Duesberg, J. C. Grunlan, G. Moriarty, J. Chen, J. Wang, A. I. Minett, V. Nicolosi and J. N. Coleman, Adv. Mater., 2011, 23, 3944.

11 Y. Li, D. Wu, Z. Zhou, C. R. Cabrera and Z. Chen, J. Phys. Chem. Lett., 2012, 3, 2221.

12 Q. H. Wang, K. Kalantar-Zadeh, A. Kis, J. N. Coleman and M. S. Strano, Nat. Nanotechnol., 2012, 7, 699.

13 M. Chhowalla, H. S. Shin, G. Eda, L.-J. Li, K. P. Loh and H. Zhang, Nat. Chem., 2013, 5, 263.

14 S. Z. Butler, S. M. Hollen, L. Cao, Y. Cui, J. A. Gupta, H. R. Gutiérrez, T. F. Heinz, S. S. Hong, J. Huang, A. F. Ismach, E. Johnston-Halperin, M. Kuno, V. V. Plashnitsa, R. D. Robinson, R. S. Ruoff, S. Salahuddin, J. Shan, L. Shi, M. G. Spencer, M. Terrones, W. Windl and J. E. Goldberger, ACS Nano, 2013, 7, 2898.

15 M. Naguib, M. Kurtoglu, V. Presser, J. Lu, J. Niu, M. Heon, L. Hultman, Y. Gogotsi and M. W. Barsoum, Adv. Mater., 2011, 23, 4248.

16 M. Naguib, O. Mashtalir, J. Carle, V. Presser, J. Lu, L. Hultman, Y. Gogotsi and M. W. Barsoum, ACS Nano, 2012, 6, 1322.

17 X. H. Wang and Y. C. Zhou, J. Mater. Sci. Technol, 2010, 26, 38. 18 Q. Tang, Z. Zhou and P. Shen, J. Am. Chem. Soc., 2012, 134, 16909.
19 J. Come, M. Naguib, P. Rozier, M. W. Barsoum, Y. Gogotsi, P. L. Taberna, M. Morcrette and P. Simon, J. Electrochem. Soc., 2012, 159, A1368.

20 M. Naguib, J. Come, B. Dyatkin, V. Presser, P.-L. Taberna, P. Simon, M. W. Barsoum and Y. Gogotsi, Electrochem. Commun., 2012, 16, 61.

21 O. Mashtalir, M. Naguib, V. N. Mochalin, Y. Dall'Agnese, M. Heon, M. W. Barsoum and Y. Gogotsi, Nat. Commun., 2013, 4, 1716.

22 M. Khazaei, M. Arai, T. Sasaki, C.-Y. Chung, N. S. Venkataramanan, M. Estili, Y. Sakka and Y. Kawazoe, Adv. Funct. Mater., 2013, 23, 2185.

23 G. Kresse and J. Furthmüller, Comput. Mater. Sci., 1996, 6, 15. 24 G. Kresse and J. Furthmüller, Phys. Rev. B: Condens. Matter Mater. Phys., 1996, 54, 11169.

25 G. Kresse and J. Hafner, Phys. Rev. B: Condens. Matter Mater. Phys., 1993, 47, 558.

26 P. E. Blöchl, Phys. Rev. B: Condens. Matter Mater. Phys., 1994, 50, 17953.

27 G. Kresse and D. Joubert, Phys. Rev. B: Condens. Matter Mater. Phys., 1999, 59, 1758.

28 J. P. Perdew, K. Burke and M. Ernzerhof, Phys. Rev. Lett., 1996, 77, 3865.

29 G. Henkelman, B. P. Uberuaga and H. Jonsson, J. Chem. Phys., 2000, 113, 9901.

30 M. S. Palmer, M. Neurock and M. M. Olken, J. Am. Chem. Soc., 2002, 124, 8452.

31 L. Verlet, Phys. Rev., 1967, 159, 98.

32 P. Mori-Sánchez, A. J. Cohen and W. Yang, Phys. Rev. Lett., 2008, 100, 146401.

33 Z. Shi, J. J. Zhang, Z. S. Liu, H. J. Wang and D. P. Wilkinson, Electrochim. Acta, 2006, 51, 1905.

34 K. P. Huber and G. Herzberg, Molecular Spectra And Molecular Structure, IV. Constants of Diatomic Molecules, Van Nostrand Reinhold, New York, 1979.

35 H. Zhang, A. Soon, B. Delley and C. Stampfl, Phys. Rev. B: Condens. Matter Mater. Phys., 2008, 78, 045436.

36 Z. Duan and G. Wang, J. Phys. Chem. C, 2013, 117, 6284.

37 Y. Xu, A. V. Ruban and M. Mavrikakis, J. Am. Chem. Soc., 2004, 126, 4717.

38 R. Bader, Atoms in Molecules: A Quantum Theory, Oxford University Press, New York, 1990.

39 Y. Choi, M. C. Lin and M. Liu, Angew. Chem., Int. Ed., 2007, 46, 7214.

40 Y. Xie and P. R. C. Kent, Phys. Rev. B: Condens. Matter Mater. Phys., 2013, 87, 235441.

41 I. J. Hsu, Y. C. Kimmel, Y. Dai, S. L. Chen and J. G. G. Chen, J. Power Sources, 2012, 199, 46.

42 M. H. Shao, B. Merzougui, K. Shoemaker, L. Stolar, L. Protsailo, Z. J. Mellinger, I. J. Hsu and J. G. G. Chen, J. Power Sources, 2011, 196, 7426.

43 W. M. Zhu, A. Ignaszak, C. J. Song, R. Baker, R. Hui, J. J. Zhang, F. H. Nan, G. Botton, S. Y. Ye and S. Campbell, Electrochim. Acta, 2012, 61, 198. 